\title{
ATAC-Seq Identifies Chromatin Landscapes Linked to the Regulation of Oxidative Stress in the Human Fungal Pathogen Candida albicans
}

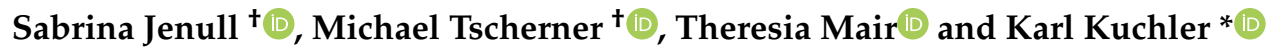 \\ Medical University of Vienna, Center for Medical Biochemistry, Max Perutz Labs Vienna, \\ Campus Vienna Biocenter, Dr. Bohr-Gasse 9/2, A-1030 Vienna, Austria; sabrina.jenull@meduniwien.ac.at (S.J.); \\ michael.tscherner@univie.ac.at (M.T.); theresia.mair@meduniwien.ac.at (T.M.) \\ * Correspondence: karl.kuchler@meduniwien.ac.at; Tel.: +43-14-2776-1807 \\ + These authors contributed equally to this paper.
}

Received: 28 August 2020; Accepted: 15 September 2020; Published: 21 September 2020

\begin{abstract}
Human fungal pathogens often encounter fungicidal stress upon host invasion, but they can swiftly adapt by transcriptional reprogramming that enables pathogen survival. Fungal immune evasion is tightly connected to chromatin regulation. Hence, fungal chromatin modifiers pose alternative treatment options to combat fungal infections. Here, we present an assay for transposase-accessible chromatin using sequencing (ATAC-seq) protocol adapted for the opportunistic pathogen Candida albicans to gain further insight into the interplay of chromatin accessibility and gene expression mounted during fungal adaptation to oxidative stress. The ATAC-seq workflow not only facilitates the robust detection of genomic regions with accessible chromatin but also allows for the precise modeling of nucleosome positions in C. albicans. Importantly, the data reveal genes with altered chromatin accessibility in upstream regulatory regions, which correlate with transcriptional regulation during oxidative stress. Interestingly, many genes show increased chromatin accessibility without change in gene expression upon stress exposure. Such chromatin signatures could predict yet unknown regulatory factors under highly dynamic transcriptional control. Additionally, de novo motif analysis in genomic regions with increased chromatin accessibility upon $\mathrm{H}_{2} \mathrm{O}_{2}$ treatment shows significant enrichment for Cap1 binding sites, a major factor of oxidative stress responses in C. albicans. Taken together, the ATAC-seq workflow enables the identification of chromatin signatures and highlights the dynamics of regulatory mechanisms mediating environmental adaptation of $C$. albicans.
\end{abstract}

Keywords: candida; chromatin; oxidative stress; ATAC-seq

\section{Introduction}

Human fungal pathogens respond to host immune defense through numerous mechanisms, including chromatin-mediated adaptive gene expression [1-3]. For example, immune defense or environmental changes can trigger pathogen responses through extracellular sensing, intracellular signal transduction, and transcriptional reprogramming [4,5]. Transcriptional changes require a tight interplay of chromatin states and transcription factors [6,7], as the swift adaptation to environmental changes is often paramount for a successful lifestyle or immune evasion. For instance, pathogens encounter a number of extreme stress conditions during the course of an infection. These include limitations in nutrient availability and the cytotoxic attack by the host immune system $[8,9]$. The opportunistic fungal pathogen Candida albicans is an extraordinary example of how a pathogen can occupy multiple host niches to persist and survive. C. albicans lives as a harmless commensal in the majority of humans, colonizing mucosal surfaces and epithelial barriers, especially the intestinal tract. 
However, severe immunodeficiency or a dysregulation of the host microbiota turns C. albicans into an invasive pathogen that can infect virtually any tissue or organ in the human body $[10,11]$. In the last decade, numerous efforts have been made to better understand fungal pathogenicity mechanisms, including the transcriptional regulation of environmental adaptation and virulence factors, such as the switch between different cellular morphologies [12-14]. Given the pivotal interplay of chromatin modifications and transcription control, it is not surprising that several C. albicans chromatin-modifying factors play important roles in fungal virulence. For instance, the functions of the histone acetyl transferases (HATs) Gcn5, Hat1, and Rtt109 are crucial for morphogenesis and virulence [15-19]. Likewise, the histone deacetylase (HDAC) complex Set3C controls transcriptional kinetics during the morphological transition from yeast growth to filamentous hyphal growth. Remarkably, genetic ablation of SET3 abrogates fungal virulence [20]. Hence, attacking chromatin modifiers provides a new option for antifungal drug development. This requires immediate attention in clinical settings, given the rapid emergence of antifungal drug resistance in Candida spp. such as C. glabrata or C. auris [17,21-25]. However, a better understanding of the interplay between chromatin architecture in the pathogen and transcriptional reprogramming during host interaction would further aid the discovery of new antifungals targeting chromatin function.

Several methods including chromatin immunoprecipitation (ChIP) and micrococcal nuclease (MNase) digestion of chromatin coupled with next-generation sequencing (ChIP-seq and MNase-seq, respectively) or DNase-seq have been employed to analyze chromatin accessibility and nucleosome positioning to reveal regulatory mechanisms [26-30]. Recently, the assay for transposase-accessible chromatin using sequencing (ATAC-seq) emerged to probe for native chromatin states, which includes accessibility as well as nucleosome positioning. ATAC-seq employs a hyperactive $\operatorname{Tn} 5$ transposase loaded with sequencing adapters, which are inserted into accessible chromatin sites, causing fragmentation and tagging of chromatin DNA referred to as tagmentation. This happens preferably at genomic regions with open, accessible chromatin, since transposition events into condensed chromatin are less likely [30,31]. Due to its technical simplicity and the low sample input requirements, ATAC-seq has been widely applied for chromatin profiling in various cell types [31-34], tissues [35,36], and even single cells $[37,38]$. Moreover, it has proved to be a useful tool for identifying sequence motifs decorated by transcriptional regulators and for predicting gene transcription [39-42].

Here, we aimed to adapt the original ATAC-seq protocol [30] for the human fungal pathogen C. albicans. We developed a modified protocol and bioinformatics workflow that enables the sensitive detection of changes in the global chromatin landscapes in response to environmental stress. We have chosen oxidative stress as environmental cue, because it triggers genome-wide changes in gene expression $[14,16]$ and because it closely mimics the oxidative immune defense fungal pathogens face during host invasion $[43,44]$. Moreover, transcriptomics data for C. albicans challenged with hydrogen peroxide $\left(\mathrm{H}_{2} \mathrm{O}_{2}\right)$ and genome-wide binding data of the oxidative stress transcriptional regulator Cap1 are available $[16,45]$, which we combined with the present ATAC-seq data for C. albicans. With this approach, we demonstrate that $\mathrm{H}_{2} \mathrm{O}_{2}$-treatment of fungal cells increases chromatin accessibility in upstream regions of genes associated with the oxidative response, and we show that those genes tend to be transcriptionally upregulated. Moreover, signatures of accessible chromatin regions enable the prediction of putative novel regulators of stress signaling that are not yet linked to transcriptional control. In addition, genomic regions with an elevated ATAC-seq signal are enriched in binding sites for the key regulator Cap1, demonstrating the potential for de novo motif discovery of regulatory factors. In summary, we show the versatility of ATAC-seq chromatin profiling in C. albicans, especially when combined with complementary next-generation sequencing data such as RNA-seq. This approach uncovers dynamic and complex regulatory mechanisms during environmental adaptation of pathogens. 


\section{Materials and Methods}

\subsection{Fungal Strains, Media, and Growth Conditions}

The C. albicans mating type-like loci (MTL) a/ $\alpha$ clinical isolate SC5314 [46] was used for all experiments and was routinely grown in YPD (1\% yeast extract, $2 \%$ peptone, $2 \%$ glucose; all BD Biosciences) at $30^{\circ} \mathrm{C}$.

To induce oxidative stress, C. albicans was first grown overnight to an optical density at $600 \mathrm{~nm}$ $\left(\mathrm{OD}_{600}\right)$ of approximately 1 in $\mathrm{YPD}$ at $30^{\circ} \mathrm{C}$ and was then treated with $1.6 \mathrm{mM} \mathrm{H}_{2} \mathrm{O}_{2}$ (Sigma-Aldrich, Burlington, MA, USA) for 15 min while shaking at $30^{\circ} \mathrm{C}$. As control, cells without $\mathrm{H}_{2} \mathrm{O}_{2}$ treatment were cultured in parallel. Cells were harvested and directly subjected to spheroplasting and tagmentation (see below).

\subsection{Nuclei and Genomic DNA (gDNA) Isolation, Tagmentation, ATAC-Seq Libraries, and Sequencing}

Nuclei preparation for tagmentation from C. albicans grown in $\mathrm{YPD}+/-\mathrm{H}_{2} \mathrm{O}_{2}$ was based on a previously published protocol used for Saccharomyces cerevisiae [31]. Briefly, $3 \times 10^{7}$ C. albicans cells were washed $1 \times$ in sorbitol buffer (1.4 M sorbitol, $40 \mathrm{mM}$ HEPES-KOH pH 7.5, $0.5 \mathrm{mM} \mathrm{MgCl}, 10 \mathrm{mM}$ DTT; all Sigma-Aldrich). After cell harvesting, the pellet was then resuspended in $330 \mu \mathrm{L}$ sorbitol buffer and $192 \mu \mathrm{L}$ of this suspension was mixed with $8 \mu \mathrm{L}$ of $50 \mathrm{mg} / \mathrm{mL} 100 \mathrm{~T}$ Zymolase $(2 \mathrm{mg} / \mathrm{mL}$ final concentration; Sigma-Aldrich) and incubated for $5 \mathrm{~min}$ at $30^{\circ} \mathrm{C}$. Spheroplasting efficiency was monitored by diluting a $10 \mu \mathrm{L}$ aliquot of the spheroplasting reaction into $1 \mathrm{~mL}$ distilled water and $\mathrm{OD}_{600}$ measurement. After $5 \mathrm{~min}$ of spheroplasting, the $\mathrm{OD}_{600}$ dropped by $>90 \%$ with respect to the initial $\mathrm{OD}_{600}$. To sustain oxidative stress for $\mathrm{H}_{2} \mathrm{O}_{2}$-treated cells, $1.6 \mathrm{mM} \mathrm{H}_{2} \mathrm{O}_{2}$ was added to the spheroplasting reaction. Hence, the total time of $\mathrm{H}_{2} \mathrm{O}_{2}$ exposure was $20 \mathrm{~min}$. Spheroplasts were then harvested for $5 \mathrm{~min}$ at $2000 \times g$ at $4{ }^{\circ} \mathrm{C}$ and washed $1 \times$ in ice-cold sorbitol buffer without DTT. The pellet was resuspended in $500 \mu \mathrm{L}$ ice-cold sorbitol buffer without DTT and spheroplasts were counted on a CASY ${ }^{\circledR}$ cell counter. Five million spheroplasts were transferred into a tube on ice, harvested for $2 \mathrm{~min}$ at $2000 \times g$ at $4{ }^{\circ} \mathrm{C}$ and the resulting cell pellet was used for subsequent tagmentation.

\subsection{1. gDNA Isolation}

To control for the sequence bias of the Tn5 transposase (TDE1) [47], gDNA was isolated via phenol-chloroform-isoamyl alcohol (PCI; Sigma-Aldrich) extraction from stationary-phase cells from C. albicans as described earlier [48] with slight modifications. Briefly, cells were broken up in lysis buffer [48] using a FastPrep instrument (MP Biomedicals, Santa Ana, CA, USA; settings: 2 rounds of $45 \mathrm{~s} 6 \mathrm{~m} / \mathrm{s}$ with $5 \mathrm{~min}$ on ice in between). After PCI extraction, gDNA was precipitated with $100 \%$ ethanol, treated with $10 \mathrm{mg} / \mathrm{mL}$ RNase A (Sigma-Aldrich) and again precipitated with ammonium acetate and 100\% ethanol. The final DNA pellet was resuspended in $50 \mu \mathrm{L}$ TE $(10 \mathrm{mM}$ Tris- $\mathrm{HCl}$ pH 8, 1 mM EDTA) and subjected to the ATAC-seq library preparation workflow described below. The concentration was measured with QuantiFluor (Promega, Madison, WI, USA) according to the manufacturer's instructions.

\subsubsection{Tagmentation}

Five million fungal spheroplasts or $0.5 \mathrm{ng}$ naked gDNA were resuspended in the tagmentation reaction mix (12.50 $\mu \mathrm{L}$ Nextera $2 \times$ TD buffer, $2.00 \mu \mathrm{L}$ Nextera TDE1 (all Illumina), $0.50 \mu \mathrm{L} 50 \times$ protease inhibitor cocktail (Roche, Basel, Switzerland), $0.25 \mu \mathrm{L}$ 1\% Digitonin (New England Biolabs, Ipswich, MA, USA, Frankfurt am Main, Germany) and $10.25 \mu \mathrm{L}$ nuclease-free distilled $\mathrm{H}_{2} \mathrm{O}$ (ThermoFisher Scientific, Shanghai, China) and incubated at $37^{\circ} \mathrm{C}$ for $30 \mathrm{~min}$. The tagmentation reaction was immediately purified using a Qiagen MiniElute PCR purification kit and elution was done in $12 \mu \mathrm{L}$ elution buffer provided by the kit. 


\subsubsection{ATAC-seq Library Amplification and Size Selection}

ATAC-seq library preparation was based on the protocol published by the Greenleaf Lab [49] with minor modifications. Briefly, prior to PCR amplification of the ATAC-seq libraries, a test qPCR was performed to determine the optimal number of amplification cycles in order to avoid size and GC base bias of the ATAC-seq library [49]. The qPCR contained the following components: $1 \mu \mathrm{L}$ tagmented DNA, $0.5 \mu \mathrm{L}$ Nextera Index primer 1 noMX and Index primer 2.1. barcode ( $25 \mu \mathrm{M}$ each), $0.1 \mu \mathrm{L} 100 \times$ SYBR green (Sigma-Aldrich, freshly diluted from a 10,000× stock), $5 \mu \mathrm{L} 2 \times$ NEBnext High-Fidelity PCR Master Mix (New England Biolabs), and $2.9 \mu \mathrm{L}$ nuclease-free distilled $\mathrm{H}_{2} \mathrm{O}$ (ThermoFisher Scientific). The qPCR was performed in a Realplex Mastercycler (Eppendorf, Hamburg, Germany) with the following cycling conditions: $72{ }^{\circ} \mathrm{C} 5 \mathrm{~min}, 98^{\circ} \mathrm{C} 30 \mathrm{~s}, 25$ cycles of $98^{\circ} \mathrm{C} 10 \mathrm{~s}, 63^{\circ} \mathrm{C} 30 \mathrm{~s}, 72^{\circ} \mathrm{C} 1 \mathrm{~min}$ and a final hold step at $10^{\circ} \mathrm{C}$. The cycle number for optimal ATAC-seq library amplification was determined as in [49]. The enrichment PCR was then performed using $10 \mu \mathrm{L}$ tagmented DNA, $2.5 \mu \mathrm{L}$ Index primer 1 noMX and Index primer 2.x. barcode ( $25 \mu \mathrm{M}$ each), $25 \mu \mathrm{L} 2 \times$ NEBnext High-Fidelity PCR Master Mix (New England Biolabs) and $10 \mu \mathrm{L}$ nuclease-free distilled $\mathrm{H}_{2} \mathrm{O}$ (ThermoFisher Scientific). The PCR reaction was then incubated in a PCR thermocycler using the following conditions: $72{ }^{\circ} \mathrm{C}$ $5 \mathrm{~min}, 98^{\circ} \mathrm{C} 30 \mathrm{~s}$, $x$ cycles (depending on the test qPCR results) of $98^{\circ} \mathrm{C} 10 \mathrm{~s}, 63{ }^{\circ} \mathrm{C} 30 \mathrm{~s}, 72{ }^{\circ} \mathrm{C}$ $1 \mathrm{~min}$, and a final hold step at $10^{\circ} \mathrm{C}$. All ATAC-seq libraries from this study were PCR amplified using 11 cycles. For multiplexing, a different Nextera Index primer 2 barcode was used for each sample. See Supplementary Table S1 for a list of Nextera PCR primers used for library amplification in this study.

The amplified ATAC-seq libraries were immediately purified and size selected with a double-sided solid-phase reversible immobilization (SPRI) approach $(0.5 \times / 1.4 \times)$ using AMPure XP beads (Beckman Coulter, Pasadena, CA, USA) according to the manufacturer's instructions. Final DNA elution was done with elution buffer provided in the Qiagen MiniElute PCR purification kit. The final ATAC-seq libraries were quantified using the fluorescent dye QuantiFluor (Promega) according to the manufacturer's manual. The yield for each ATAC-seq library sample was between 60 and $90 \mathrm{nM}$ for replicate 1, replicate 2 and around $13 \mathrm{nM}$ for replicate 3 .

The quality of purified libraries was analyzed on a Bioanalyzer High Sensitivity DNA chip (Agilent, Santa Clara, CA, USA; see Figure 1B for an example) by following the manufacturer's instructions.

\subsubsection{Next-Generation Sequencing}

ATAC-seq libraries were prepared from three biological replicates for each condition (YPD, YPD $+\mathrm{H}_{2} \mathrm{O}_{2}$, and gDNA) and pooled in equimolar ratios for sequencing. Sequencing was done in 75 bp paired-end read mode on a HiSeq 3000/4000 system at the Biomedical Sequencing Facility (BSF; https:/cemm.at/research/facilities/biomedical-sequencing-facility-bsf/) at the Center of Molecular Medicine (CeMM) in Vienna, Austria.

\subsection{ATAC-Seq Data Analysis Workflow}

\subsubsection{Pre-Processing and Read Alignment}

Quality control of raw sequencing read files (.bam) was done using fastQC v0.11.8 [50]. Illumina TrueSeq adapter trimming was done via cutadapt v1.18 (https://cutadapt.readthedocs.io/en/stable/; settings: -interleaved-q $30-\mathrm{O} 1$ ). Trimmed reads were then aligned to the haploid $C$. albicans SC5314 genome (assembly 22, version A22-s07-m01-r88; http://www.candidagenome.org/) using NextGenMap v0.5.5 [51] only keeping aligned reads with a minimum mapping quality of 30 (settings: - b -p -Q 30). Optical read duplicates were removed using Picard tools (Broad Institute, https://broadinstitute.github.io/picard/, settings: MarkDuplicates REMOVE_DUPLICATES=true VALIDATION_STRINGENCY=LENIENT; Broad Institute, https://broadinstitute.github.io/picard/) and mitochondrial reads were removed using the "intersect" tool from BEDTools with -v settings (https://github.com/arq5x/bedtools2). The average number of mapped reads per conditions for each 
chromosome and the size of each chromosome in comparison are shown in Supplementary Figure S1. Chromosome sizes of C. albicans were retrieved from the National Center for Biotechnology Information (NCBI). The ATAC-seq fragment length distribution from properly paired reads is represented in Figure 1C.

A

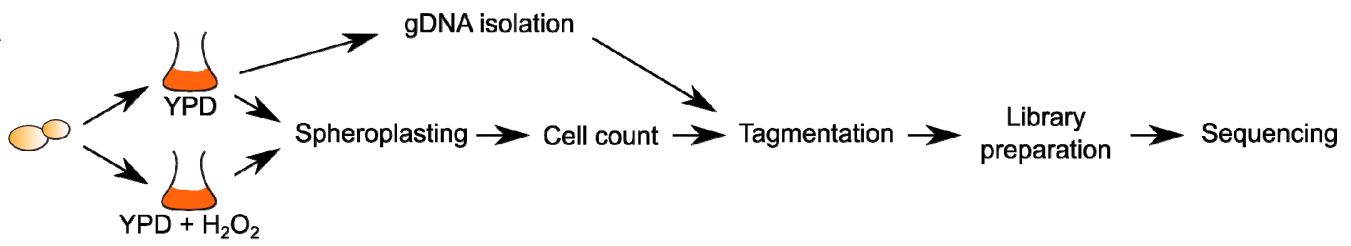

B
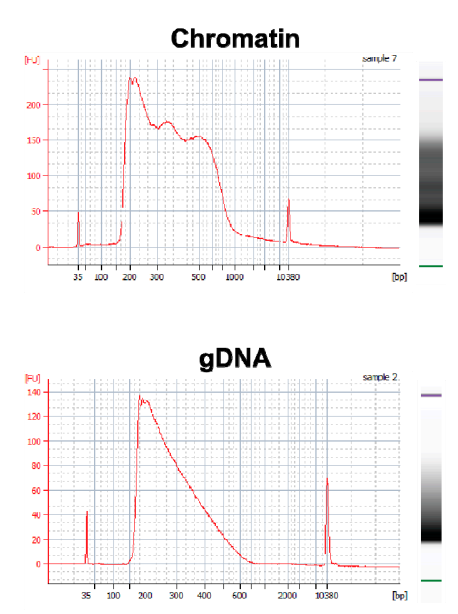

C gDNA

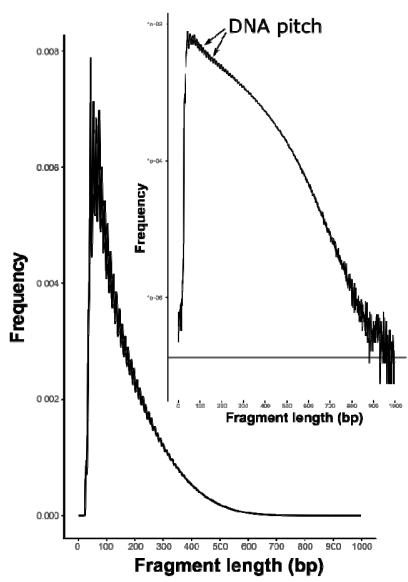

Chromatin

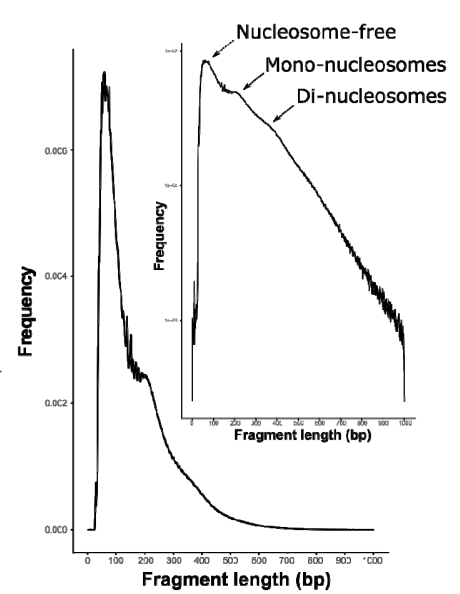

D

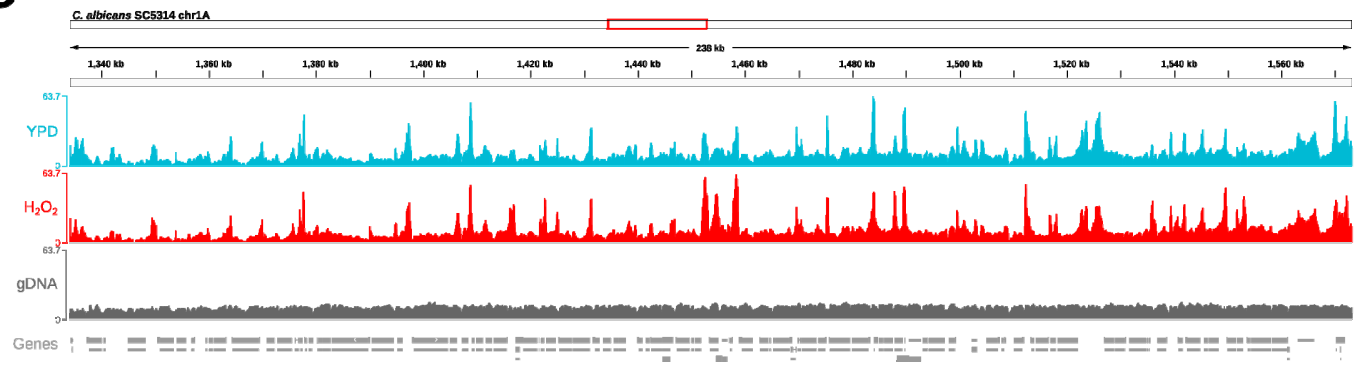

Figure 1. Quality control of assay for transposase-accessible chromatin using sequencing (ATAC-seq) libraries from Candida albicans treated or non-treated with $\mathrm{H}_{2} \mathrm{O}_{2}$. (A) Experimental set-up for ATAC-seq profiling of $5 \times 10^{6}$ C. albicans (see Materials and Methods for details). (B) Bioanalyzer electrophoretic profiles and gel image from ATAC-seq libraries prepared from fungal chromatin (isolated nuclei) or naked gDNA. The x-axis from the electropherogram depicts the fragment size distribution (bp) of tagmented samples and the $y$-axis represents the abundance. A distinct nucleosomal pattern (mono-, di-, and tri-nucleosomes) is visible in chromatin samples, but not in tagmented gDNA samples. (C) Fragment length distribution of ATAC-seq reads from gDNA and nuclei prepared from YPD (1\% yeast extract, $2 \%$ peptone, $2 \%$ glucose)-grown cells. The fragment length in bp (x-axis) from one representative biological replicate from each group is plotted against its frequency (y-axis). The graph insert shows the log-transformed histogram. Signals originating from the DNA helical pitch [31] are emphasized by arrows on the gDNA plot. Arrows on the chromatin plot indicate nucleosome-free ATAC-seq read fragments and nucleosome occupied read fragments. (D) Integrative Genomics Viewer (IGV) browser snap-shot from a $238 \mathrm{~kb}$ region of the C. albicans chromosome 1A of ATAC-seq reads from aligned BAM files. The biological replicates from each sample group were pooled. Genes are depicted as gray boxes below the sequencing read coverage tracks. 
Aligned BAM files were split according to the fragment lengths of the sequencing read pairs as done previously [30]. Read fragments below $100 \mathrm{bp}$ were considered as coming from nucleosome-free regions and were used for further analysis. Normalized read coverage files (bigWig) of nucleosome-free read fragments were created using deepTools2 "bamCoverage" ([52]; settings: -e -bs 5 -normalizeUsing $\mathrm{CPM}$ ) and visualized using the Integrative Genomics Viewer (IGV; [53]).

\subsubsection{Prediction of Nucleosomal Positions and Genomic Coverage of ATAC-seq Signals}

Nucleosomal occupancies were predicted using NucleoATAC [31] with default parameters. NucleoATAC analysis was performed for all C. albicans promoter regions (transcription start site (TSS) -/+ 1000 bp). Promoter regions were extracted using the "promoters" function from the Bioconductor GenomicRanges package [54]. The NucleoATAC bedgraph output files were further converted to the bigWig format using the bedGraphToBigWig tool (https://github.com/ENCODE-DCC/kentUtils). The NucleoATAC result was further compared with the nucleosome-free ATAC-seq reads (see above) and published MNase-seq data for C. albicans grown in YPD [55]. MNase-seq raw datasets were downloaded via sequence read archive (SRA) and processed as the ATAC-seq raw data. The aligned BAM files were converted into a normalized read coverage (bigWig) file using the deepTools2 "bamCoverage" function ([52], settings: as above, except -bs 1). Genomic read coverage tracks were visualized with the IGV. To plot the read coverage of nucleosome-free ATAC-seq peaks, nucleosome positions from the NucleoATAC analysis, and the published MNase-seq data [55] over all C. albicans transcripts, a coverage matrix was computed using the deepTools2 "computeMatrix" function ([52]; reference-point -a 1000 -b 1000 -bs 5). Transcripts from C. albicans were extracted by the "transcripts" function of the GenomicRanges package [54] using the current genomic annotation from the C. albicans assembly 22 (version A22-s07-m01-r88; http://www.candidagenome.org/). MNase-seq reads were extended to $100 \mathrm{bp}$ prior to coverage plotting as described in the original publication [55].

Differential read coverage (log2-ratio) in nucleosome-free ATAC-seq peaks between $\mathrm{H}_{2} \mathrm{O}_{2}$-treated and non-treated samples was analyzed by the deepTools2 "bamCompare" function ([52]; settings: -normalizeUsing CPM -bs 5 -e-scaleFactorsMethod None). For downstream analysis, this output was first used to compute a coverage matrix with the deepTools2 "computeMatrix" tool [52] as described above across promoter regions $(-1000 /+200 \mathrm{bp}$ with respect to the TSS) of all C. albicans transcripts. Heat map and coverage plots were generated using the "plotHeatmap" function from deepTools2 [52] using K-means clustering (settings: -kmeans 4). The full analysis data and the RNA-seq regulation of clustered genes are provided in Supplementary Table S2. Coverage regions from cluster 1 and cluster 4 were further subjected to gene ontology (GO) term analysis using the "enrichGO" function from the clusterProfiler package ([56]; settings: ont $=$ "BP", qvalueCutoff $=0.05$, readable $=$ TRUE). The GO term analysis result was plotted using the clusterProfiler "dotplot" function after merging redundant GO terms using the "simplify" function from the same package (settings: cutoff $=0.7$ ) [56].

\subsubsection{Peak Calling and Genomic Annotation of ATAC-seq Peaks}

Peak-calling for each individual sample was done with MACS2 v2.1.2 using "callpeak" ([57]; settings: -f BAMPE -g 14521502). The aligned read fragments from the gDNA ATAC-seq libraries were merged into one BAM file using the SAMtools "merge" function [58] and used as a background control for peak calling. Peaks from all samples and replicates were merged and converted to the GFF file format for read counting using htseq-count ([59]; settings: -f bam-s no-t peak) and differential ATAC-seq peak analysis (see below). The reproducibility of called ATAC-seq peaks among biological replicates was analyzed via principal component analysis (PCA) using the "prcomp" function from the $\mathrm{R}$ stats package (https://www.rdocumentation.org/packages/stats). For the ATAC-seq peak annotation, the genomic annotation from C. albicans assembly 22 (version A22-s07-m01-r88) was downloaded from the Candia Genome Database (CGD; http://www.candidagenome.org/) and was merged with the $5^{\prime}$ UTR (untranslated region) and TSS annotations of $C$. albicans, which were retrieved from the yeast transcription start site database (YeasTSS) (http://www.yeastss.org/). This file was further used to create 
a TxDb object using the GenomicFeatures "makeTxDbFromGFF" function ([54]; settings: dataSource = “CGD”, organism = "Candida albicans SC5314", circ_seqs = "Ca22chrM_C_albicans_SC5314"). Peak annotation was done using the ChIPseeker Bioconductor package [60] with the "annotatePeak" function (options: tssRegion $=c(-2000,0)$, genomicAnnotationPriority $=c($ "Promoter", "5UTR", "3UTR", "Exon", "Intron", “Downstream", "Intergenic")). The distribution and overlaps of peak annotations among genomic features were visualized with the "upsetplot" function from the ChIPseeker package. The ATAC-seq peak location across all chromosomes and the fold change in peak signals between $\mathrm{H}_{2} \mathrm{O}_{2}$-treated and non-treated samples were visualized using the karyoploteR package [61].

\subsubsection{Analysis of Differential ATAC-seq Peaks}

Differential accessible peak detection was done using the edgeR package [62] with the generalized linear model (GLM) approach. Since replicate three clustered away from the other two replicates in the PCA analysis (Supplementary Figure S3), the GLM approach was combined with batch effect correction. Supplementary Table S3 contains a complete list of detected ATAC-seq peaks and the edgeR analysis result. Supplementary Table S4 presents the ATAC-seq results overlaid by the RNA-seq datasets. GO term analysis of genes with significantly increased peak signal (log2-fold change $>0$, false discovery rate $(F D R)<0.05)$ in upstream regions upon $\mathrm{H}_{2} \mathrm{O}_{2}$ stress was performed with the "enrichGO" function from the clusterProfiler package [56] as described above.

\subsubsection{Motif Search}

Nucleosome-free ATAC-seq peaks with increased signal in the $\mathrm{H}_{2} \mathrm{O}_{2}$-treated samples (log2-fold change $>0$, FDR $<0.05)$ from the edgeR analysis were further subjected to motif search analysis using the MEME suite (v5.1.0; [63]; http://meme-suite.org/). First, the previously identified C. albicans Cap1 regulon [45] was used to generate the position weight matrix for Cap1 with the MEME web application (settings: 1 occurrence per sequence, motif width $=8$ ). This was further used as input for the MEME FIMO tool [64] to analyze Cap1 binding sites in the upregulated nucleosome-free ATAC-seq peaks in $\mathrm{H}_{2} \mathrm{O}_{2}$-treated samples (settings: -parse-genomic-coord). The enrichment of the identified Cap1 binding sites was re-formatted to the BED file format and further used to compute a coverage matrix with the "computeMatrix" function from deepTools2 ([52]; settings: reference-point, -b 500 -a 500 -bs 5 -referencePoint center) in nucleosome-free ATAC-seq peaks from $\mathrm{H}_{2} \mathrm{O}_{2}$-treated and non-treated samples. For de novo motif analysis, upregulated nucleosome-free ATAC-seq peaks during oxidative stress were filtered based on their occurrence within the $1 \mathrm{~kb}$ upstream region of a gene and $500 \mathrm{bp}$ of maximum peak width. The resulting 184 regulated peaks were used for de novo motif search using the MEME suite online tool DREME ([65]; parameters: E-value threshold 1; search given strand only).

\subsubsection{Data Plotting}

Unless otherwise stated, plots were done with the "ggplot2" package in R [66].

\subsection{External Datasets}

Our previously published [16] RNA-seq data from C. albicans in response to $30 \mathrm{~min}_{2} \mathrm{H}_{2}$ treatment (Gene Expression Omnibus (GEO) accession number GSE73409) were used to compare ATAC-seq peaks with increased signal in $\mathrm{H}_{2} \mathrm{O}_{2}$-treated samples with genes upregulated in response to oxidative stress. MNase-seq data ([55]; described above) was downloaded from the SRA (SRR059732).

\subsection{Code Availability}

The entire bioinformatics analysis pipeline is freely accessible on github: https://github.com/ tschemic/ATACseq_analysis. 


\subsection{Data Availability}

ATAC-seq data have been deposited at the GEO under the accession number GSE156582.

\section{Results and Discussion}

\subsection{ATAC-Seq in C. albicans Reflects Nucleosomal Organization Genome-Wide}

We adapted and optimized an ATAC-seq method originally developed for the fungi S. cerevisiae and Schizosaccharomyces pombe [31] to study alterations in chromatin landscapes upon oxidative stress in the human fungal pathogen C. albicans. Therefore, logarithmically growing C. albicans cells were either treated with $1.6 \mathrm{mM} \mathrm{H}_{2} \mathrm{O}_{2}$ for $15 \mathrm{~min}$ or left untreated (see Materials and Methods for details). After spheroplasting, cells were then subjected to sample preparation for ATAC-seq analysis. To correct for the known sequence bias of the Tn5 transposase [47], naked genomic DNA (gDNA) prepared from YPD-grown C. albicans was included in the workflow (Figure 1A; see Materials and Methods for details). Three biological replicates were prepared for each condition. Analysis of PCR-amplified ATAC-seq libraries from intact fungal cell nuclei (chromatin) showed a distinct fragment size distribution reminiscent of nucleosomal periodicity. Importantly, ATAC-seq libraries prepared from naked gDNA had a less complex fragment distribution as they lacked nucleosomal patterns (Figure 1B). The same trend was observed after $75 \mathrm{bp}$ paired-end sequencing (PES), where the fragment length distribution of PES reads from fungal chromatin showed a predominant peak of shorter fragments around $<150 \mathrm{bp}$, representing putative nucleosome-free regions. Another peak with an average fragment length of $200 \mathrm{bp}$ reflected DNA regions occupied by one nucleosome (Figure 1C; [49]). Additionally, a weak enrichment for di-nucleosomal peaks was detected. Again, ATAC-seq libraries from gDNA did not reveal nucleosomal presence (Figure 1C). Instead, the majority of PES read fragments were below $150 \mathrm{bp}$, which was consistent with the fragment length distribution of Tn5-generated DNA sequencing libraries [67]. Notably, the number of mapped reads was evenly distributed among the $C$. albicans chromosomes, thus reflecting total chromosome sizes and suggesting no chromosomal bias (Supplementary Figure S1A,B). Inspection of ATAC-seq reads aligned to the C. albicans genome further revealed a distinct read coverage profile of accessible chromatin regions in nuclear chromatin purified from $\mathrm{H}_{2} \mathrm{O}_{2}$-treated $\left(\mathrm{H}_{2} \mathrm{O}_{2}\right)$ and non-treated (YPD) cells, which was not apparent in gDNA libraries (Figure 1D shows a region of chromosome 1 as an example).

To further test the robustness of our ATAC-seq workflow, we first selected ATAC-seq PES read fragments below $100 \mathrm{bp}$, which correspond to putative nucleosome-free genomic regions [30]. These data were subjected to nucleosomal occupancy prediction using the NucleoATAC tool [31]. First, read coverage profiles of nucleosome-free ATAC-seq peaks and NucleoATAC-called nucleosomes were inspected at an example locus on chromosome 6 containing four open reading frames (ORFs) (Figure 2A). Pronounced nucleosome-free ATAC-seq peaks, flanked by nucleosomal signals as predicted by NucleoATAC, were detected in upstream promoter regions (Figure 2A, black arrows).

Moreover, nucleosomal positions predicted by NucleoATAC correlated very well with published C. albicans MNase-seq data [55] (Figure 2B). By averaging the read coverage of nucleosome-free and mononucleosmal ATAC-seq signals across all C. albicans transcripts relative to the transcription start site (TSS), we further observed an enrichment of nucleosome-free ATAC-seq reads adjacent to the TSS. Mononucleosomal ATAC-seq signals were enriched up- and downstream of the nucleosome-free peak, reflecting a well-positioned +1 nucleosome and a typical nucleosomal organization of canonical active gene promoters [26,68] (Figure 2B). Taken together, ATAC-seq libraries generated from C. albicans native chromatin share typical features of mammalian and other fungal (S. cerevisiae and Schizosaccharomyces pombe) ATAC-seq samples [30,31]. Hence, the approach is suitable to probe chromatin accessibility and nucleosomal positioning in C. albicans and their changes during stress response. 
A

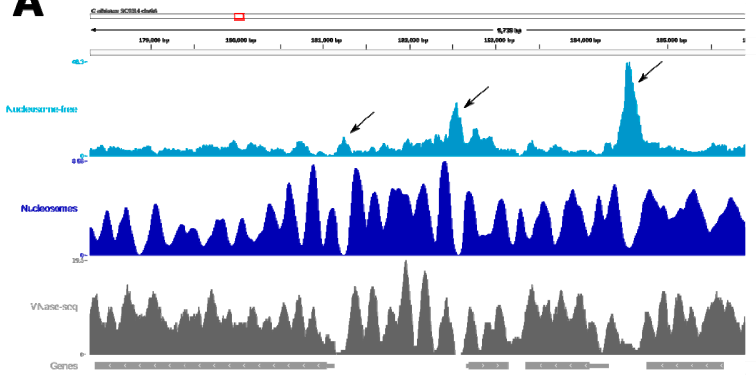

B

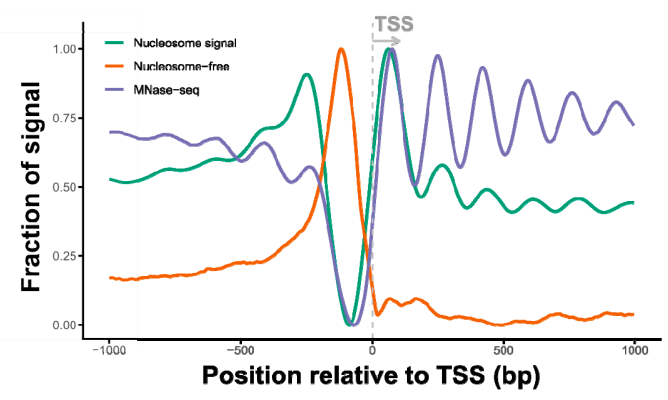

C

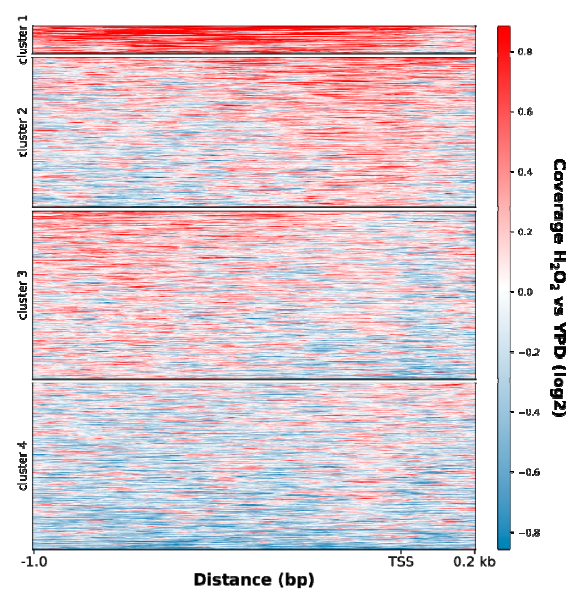

D

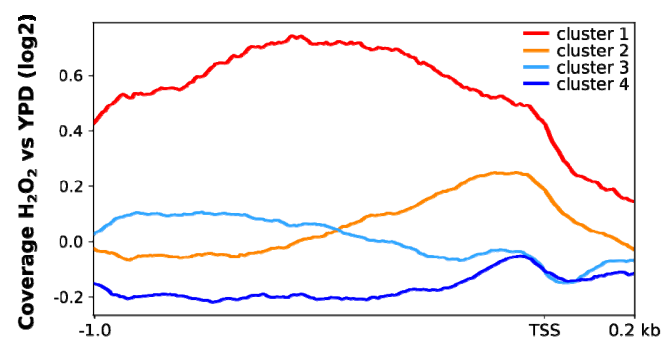

E

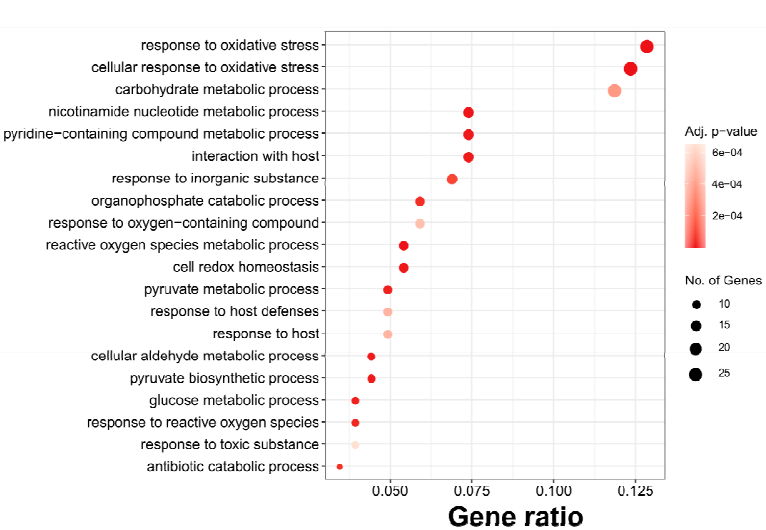

$\mathbf{F}$

Response to oxidative stress $\log 2 \mathrm{FC}$ RNA-seq $\begin{array}{llll}6 & 4 & 2 & 0\end{array}$

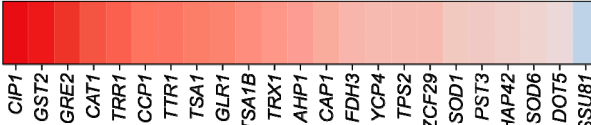

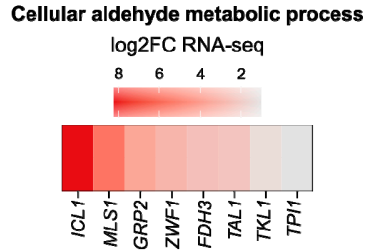

Cellular aldehyde metabolic process log2FC RNA-seq $\begin{array}{llll}8 & 6 & 4 & 2\end{array}$ og2FC RNA-seq $\begin{array}{llllll}6 & 5 & 4 & 3 & 2 & 1\end{array}$

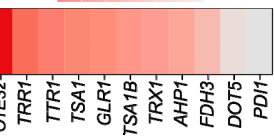

Figure 2. Chromatin accessibility is increased at promoters associated with oxidative stress genes. (A,B) ATAC-seq analysis in C. albicans allows for nucleosomal occupancy prediction. Comparison of coverage profiles of nucleosome-free ATAC-seq read signals with mono-nucleosomal position prediction and MNase-seq data [55] at a region on chromosome 6 (A) or across all C. albicans transcriptional start sites (+/-1000 bp relative to the transcription start site (TSS)) (B). Data represent ATAC-seq samples from YPD grown cells and pooled biological replicates (see Materials and Methods for details). In panel A, genes are depicted as gray boxes with white arrows indicating the direction of transcription. The black arrows in the top IGV track indicate nucleosome-free ATAC-seq signals flanked by nucleosomes. (C,D) $\mathrm{K}$-means clustering of differential ATAC-seq nucleosome-free read coverage (log2-fold change) of $\mathrm{H}_{2} \mathrm{O}_{2}$-treated $\left(\mathrm{H}_{2} \mathrm{O}_{2}\right)$ vs. non-treated (YPD) cells represented as heat maps (C) or read coverage profiles across all C. albicans promoters $(-1000 \mathrm{bp} /+200 \mathrm{bp}$ relative to the TSS). (E,F) Gene ontology (GO) term analysis of cluster 1 from panel $C$ and $D$ represented as a dot plot $(\mathbf{E})$ and heat maps $(\mathbf{F})$. Genomic regions represented in cluster 1 were annotated to the next downstream gene. The gene ratio indicates the proportion of genes enriched in this GO term relative to input genes. The color scale indicates the adjusted $p$-value of the GO enrichment analysis. Heat maps show genes from the indicated GO term category with the color scale representing the log2-fold change in transcription (from RNA-seq data) in response to oxidative stress. 


\subsection{ATAC-Seq Detects Genome-Wide Changes in Chromatin Accessibility after Oxidative Stress}

Next, we aimed to assess whether ATAC-seq in C. albicans is able to measure changes in chromatin accessibility at genomic regions associated with the oxidative stress response. Therefore, we analyzed the differential read coverage of nucleosome-free ATAC-seq peaks in $\mathrm{H}_{2} \mathrm{O}_{2}$-treated and non-treated cells. Then, we further clustered all C. albicans promoter regions $(-1000 /+200 \mathrm{bp}$ upstream of the TSS) based on their differential read coverage profile using K-means clustering (Figure 2C). Genomic regions with a moderate increase in nucleosome-free ATAC-seq peak signals around the TSS were enriched in cluster 2, while cluster 3 showed a subtle increase in ATAC-seq read coverage more distal relative to the TSS (Figure 2C,D, orange and light blue lines, respectively, in panel D). In response to oxidative stress, the most striking increase in chromatin accessibility (i.e., increased nucleosome-free ATAC-seq reads) proximal to the TSS and at least $1 \mathrm{~kb}$ upstream of the TSS, was observed for loci contained in cluster 1 (Figure 2C,D, red line in panel D). GO term enrichment analysis of promoter-associated genes from cluster 1 showed that around $12 \%$ of the genes in cluster 1 are related to the oxidative stress response (Figure 2E). Cluster 4 included regions with decreased chromatin accessibility upstream of the TSS in response to $\mathrm{H}_{2} \mathrm{O}_{2}$ treatment (Figure 2C,D, dark blue line in panel D). Genes from this cluster were involved in ribosome- and translation-related processes (Supplementary Figure S2A), most of which are often downregulated during fungal stress adaptation [69-73].

The degree of chromatin accessibility is typical for genomic regions experiencing distinct transcriptional activities. For instance, the TSS of active gene promoters and enhancers is associated with increased chromatin accessibility relative to inactive elements and heterochromatic regions [28,74-76]. Hence, the combination of ATAC-seq and RNA-seq can provide highly useful insights into the chronological dynamics of changing chromatin accessibility and subsequent transcriptional responses [42,77]. In a previous study, we performed RNA-seq analysis in C. albicans in response to $\mathrm{H}_{2} \mathrm{O}_{2}$ treatment [16]. For RNA-seq, cells were treated with $1.6 \mathrm{mM} \mathrm{H}_{2} \mathrm{O}_{2}$ for $30 \mathrm{~min}$, while cells for ATAC-seq were exposed to $1.6 \mathrm{mM} \mathrm{H}_{2} \mathrm{O}_{2}$ only for a total of $20 \mathrm{~min}$. Therefore, we used the RNA-seq dataset to assess the chronological dynamics of chromatin accessibility and transcription.

When assessing the log2-fold change from our RNA-seq dataset of genes with increased chromatin accessibility upon $\mathrm{H}_{2} \mathrm{O}_{2}$ treatment in upstream genomic regions (cluster 1), we observed that transcription of the majority of cluster 1 genes was also upregulated in response to $\mathrm{H}_{2} \mathrm{O}_{2}-321$ genes mapped to cluster 1, of which 309 were also present in the RNA-seq dataset. Out of those 309 genes, 200 were transcriptionally upregulated upon $\mathrm{H}_{2} \mathrm{O}_{2}$ treatment (Supplementary Table S2). For instance, CAT1, which encodes the $\mathrm{H}_{2} \mathrm{O}_{2}$-detoxifying catalase [78], as well as the oxidative stress-related Cap1 transcription factor and its known targets OYE32 and CIP1 [79], were among genes showing remarkably increased chromatin accessibility and transcriptional induction in $\mathrm{H}_{2} \mathrm{O}_{2}$-treated cells (Figure 2F). In addition, ICL1 and MSL1 encoding for glyoxylate cycle enzymes [80] showed increased ATAC-seq peak signals, reflecting their robust transcriptional induction upon oxidative stress (Figure 2F). Similarly, genes with decreased ATAC-seq read coverage upstream of their TSS were likewise repressed upon stress (Supplementary Figure S2B). Hence, changes in ATAC-seq read coverage are predictive of alterations in gene expression.

To further dissect significant changes in chromatin accessibility, nucleosome-free ATAC-seq signals were subjected to peak calling with ATAC-seq libraries from gDNA as background noise control (see Material and Methods for details). The genomic locations of the majority of called nucleosome-free ATAC-seq peaks were located within gene promoters, exons and $5^{\prime}$ untranslated regions (UTRs) (Figure 3A) reflecting a usual distribution of genomic features among called ATAC-seq peaks [81]. 
A

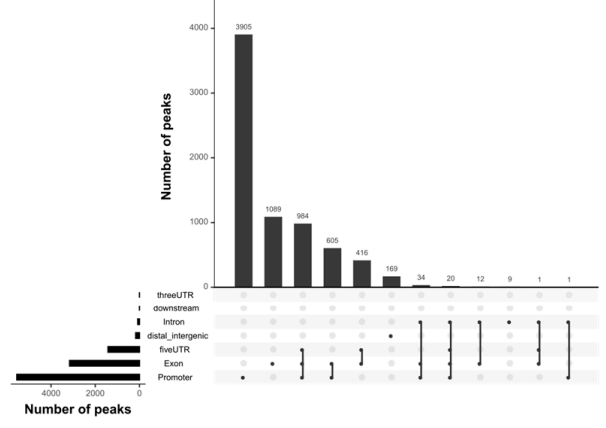

B

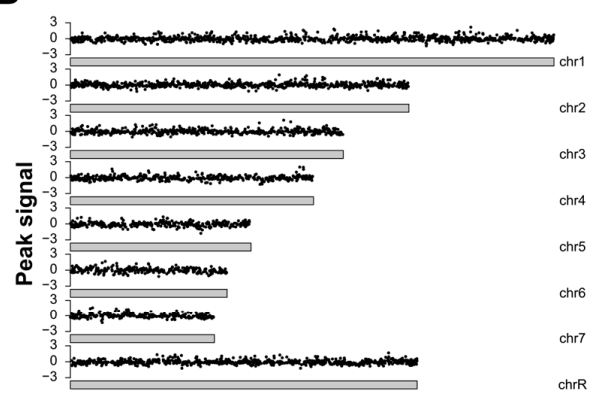

C

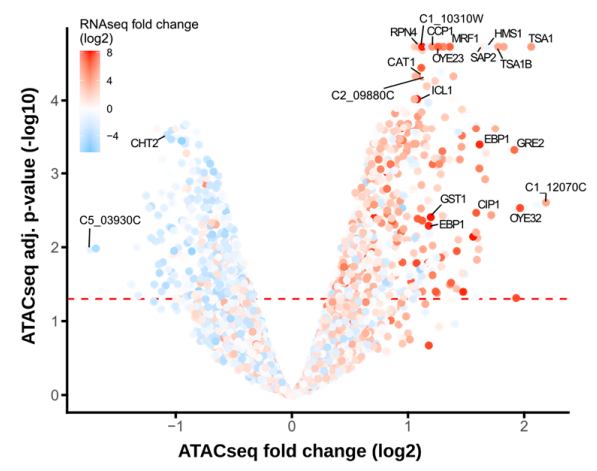

D

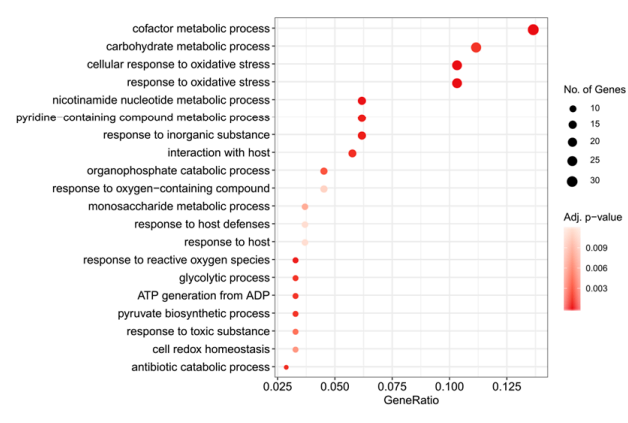

Figure 3. ATAC-seq nucleosome-free peak annotation and differential ATAC-seq peak analysis. (A) The majority of called ATAC-seq nucleosome-free peaks are within gene promoters. The vertical bars of the UpSet plot represent the number of peaks spanning one or more specific genomic regions (overlaps of genomic regions are indicated by connection lines between the black dots). For instance, the first bar indicates the number of peaks occurring exclusively in promoters, while the third bar represents peaks spanning promoter, $5^{\prime}$ UTR (untranslated regions) and exonic regions. The horizontal bars show the total number of peaks for each genomic annotation, independent of overlaps. (B) Karyoplots showing the genomic peak location on each chromosome of differential ATAC-seq nucleosome-free read signals ( $\log 2$-fold change) of $\mathrm{H}_{2} \mathrm{O}_{2}$-treated vs. non-treated cells. The log2-fold change is depicted on the $\mathrm{y}$-axis and the $\mathrm{x}$-axis represents each chromosome of the C. albicans genome. (C) Increased ATAC-seq nucleosome-free peak signals correlate with increased transcript levels. Volcano plot depicting the differential peak signal analysis of nucleosome-free ATAC-seq peak signals between $\mathrm{H}_{2} \mathrm{O}_{2}$-treated and non-treated cells. The y-axis represents the negative $\log 10$ adjusted $p$-value (false discovery rate, FDR) and the x-axis depicts the $\log 2$ fold change between $\mathrm{H}_{2} \mathrm{O}_{2}$ treatment vs. no treatment. The horizontal dashed red line indicates an FDR of 0.05. Each dot represents one peak annotated to the next downstream gene, which is overlaid with a color gradient indicating the log2-fold change $\left(\mathrm{H}_{2} \mathrm{O}_{2}\right.$ treatment vs. no treatment) from RNA-seq data. (D) Peaks with increased ATAC-seq nucleosome-free signals in $\mathrm{H}_{2} \mathrm{O}_{2}$-treated cells are upstream of genes related to the oxidative stress response. The gene ratio indicates the proportion of genes enriched in the indicated $\mathrm{GO}$ term relative to input genes with increased chromatin accessibility after $\mathrm{H}_{2} \mathrm{O}_{2}$ stress. The color scale represents the adjusted $p$-values of the GO enrichment analysis.

Of note, substantial peak overlaps between these three genomic regions were observed, indicating peaks stretching from promoter regions into the $5^{\prime}$ UTRs and the first exon. Interestingly, a previous study observed ATAC-seq signals accumulating towards sub-telomeric regions [39]. Thus, we further inspected the genome-wide distribution of differentially enriched peaks, but we did not detect a bias toward specific chromosomal regions including telomeres (Figure 3B).

Nucleosome-free ATAC-seq peaks in $\mathrm{H}_{2} \mathrm{O}_{2}$-treated and non-treated cells from all three biological replicates were then subjected to principal component analysis (PCA) based on the presence and absence of called peaks. Samples clustered according to their growth conditions, suggesting that the 
peak calling of the ATAC-seq workflow was able to identify alterations in chromatin accessibility in response to oxidative stress (Supplementary Figure S3). Notably, the PCA also revealed variations among biological replicates, as replicate three from stressed and non-stressed cells was separated from the other two replicates by the first principal component (Supplementary Figure S3, PC1). Hence, we also applied a batch effect correction during differential peak analysis of called ATAC-seq peaks (see Material and Methods for details). In total, 1092 nucleosome-free ATAC-seq peaks with significant signal alterations (FDR < 0.05) in response to oxidative stress were detected (Supplementary Table S3). Peaks were annotated to the next closest downstream gene and the resulting dataset was overlaid with the RNA-seq data to correlate differentially regulated ATAC-seq peaks with transcriptional changes. In total, 468 ATAC-seq peaks were upregulated in $\mathrm{H}_{2} \mathrm{O}_{2}$-treated cells and located within the $2 \mathrm{~kb}$ upstream region of the annotated gene, with 298 peaks detected upstream of genes with increased transcription after oxidative stress (Figure 3C, Supplementary Table S4). These data demonstrate that more than $60 \%$ of genomic regions showing increased nucleosome-free ATAC-seq peak signals within a $2 \mathrm{~kb}$ upstream range were associated with transcriptional induction. For instance, as already observed by comparing ATAC-seq read coverages between the treatment groups, oxidative stress altered chromatin accessibility of promoter regions and transcript induction of genes including CAT1, ICL1, OYE32, and TSA1, the latter encoding an antioxidative protein [82] (Figure 3C). Accordingly, genes downstream of upregulated ATAC-seq peaks represented biological processes involving oxidative stress responses. Interestingly, gene promoters of almost 90 genes, including some encoding uncharacterized proteins, displayed significantly elevated chromatin accessibility in promoter regions during $\mathrm{H}_{2} \mathrm{O}_{2}$ treatment without alterations in gene expression. For example, gene $\mathrm{C2} \_09880 \mathrm{C}$, encoding for a putative DNA-binding factor, was among the top 20 genes with increased chromatin accessibility upon oxidative stress. However, no significant alteration in transcription was detected in the corresponding RNA-seq dataset (Figure 3C and Supplementary Table S5). Such cases could represent putative regulators that are primed for transcriptional control in response to additional signaling inputs [83]. Generally, out of a total of 5284 ATAC-seq peaks detected within the $2 \mathrm{~kb}$ upstream regions of annotated genes, 3174 peaks showed no ATAC-seq signal alterations in response to oxidative stress, despite significant transcriptional regulation of the downstream gene after $\mathrm{H}_{2} \mathrm{O}_{2}$ treatment (Supplementary Table S4). These discrepancies might reflect distinct regulatory dynamics between the establishment of permissive open chromatin and highly transient transcriptional responses often seen in stress adaptation [72]. Indeed, the samples for RNA-seq data were collected after $30 \mathrm{~min}$ of oxidative stress, while the ATAC-seq dataset was derived from cells exposed to $\mathrm{H}_{2} \mathrm{O}_{2}$ for a total of $20 \mathrm{~min}$. In summary, these data demonstrate that ATAC-seq detects changes in chromatin accessibility linked to gene expression control during fungal stress adaptation. Moreover, it reflects highly dynamic transcriptional responses and could be used to predict novel regulators that are primed for further transcriptional alterations during environmental adaptation.

\subsection{Oxidative Stress-Responsive ATAC-Seq Peaks Are Enriched for Cap1 Binding Sites}

Despite assessing the chromatin architecture at different genomic features, ATAC-seq has been used to detect sequence motifs used by transcriptional regulators in ATAC-seq peak regions [32,39]. This is of special interest for the identification of as-yet-unknown transcriptional regulators or even regulatory networks governing transcriptional responses. Indeed, regulatory sequences were identified in the malaria parasite Plasmodium falciparum during intra-erythrocytic development [39]. Hence, ATAC-seq can yield new insights into transcriptional networks and their dynamic behavior during environmental adaptations. For example, major virulence traits of $C$. albicans, such as morphological transitions, are under the control of multi-layer genetic regulatory networks engaging both transcription factors and chromatin modifiers $[20,84,85]$. Hence, we tested whether our ATAC-seq data were suitable for motif discovery in C. albicans. As mentioned briefly above, the Cap1 transcription factor is the key driver of transcriptional induction during the oxidative stress response [79]. Its cis-acting sequence motif emerged from an earlier study [45] (Figure 4A). 
A

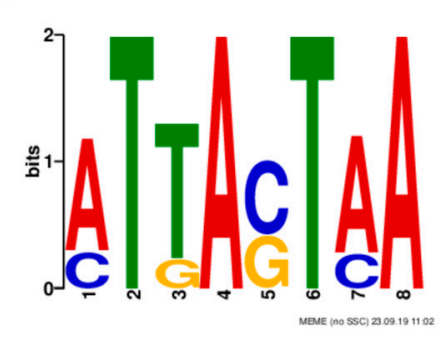

C
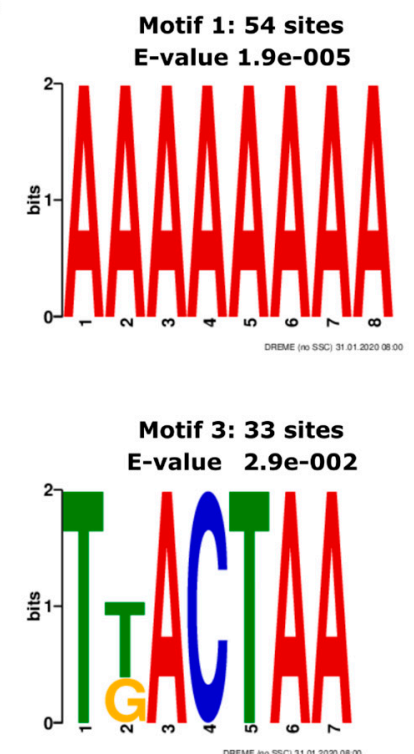

B
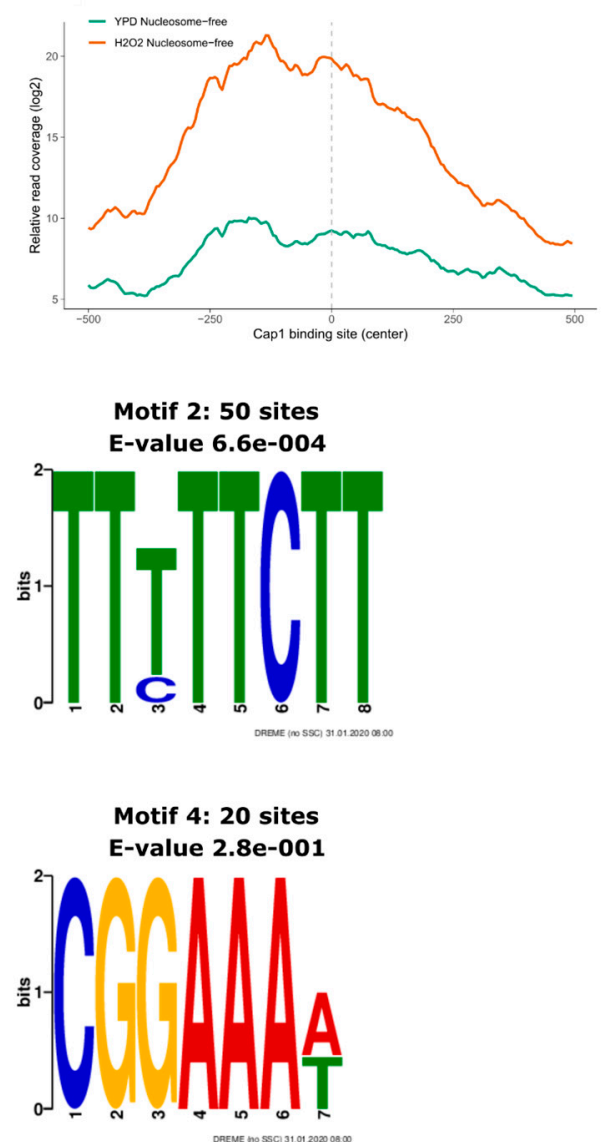

Figure 4. De novo motif search in oxidative stress-responsive nucleosome-free ATAC-seq peaks. (A) Previously identified Cap1 motif [45] used for enrichment analysis in nucleosome-free ATAC-seq peaks with increased signal in $\mathrm{H}_{2} \mathrm{O}_{2}$-treated cells. (B) Cap1 binding sites are enriched for nucleosome-free ATAC-seq fragments in response to $\mathrm{H}_{2} \mathrm{O}_{2}$. Published Cap1 binding sites [45] were used for enrichment analysis within nucleosome-free ATAC-seq peaks. Read coverage in $\mathrm{H}_{2} \mathrm{O}_{2}$-treated and untreated cells around the Cap1 binding sites is shown. (C) Logos of position weight matrices from de novo identified motifs occurring in nucleosome-free ATAC-seq peaks in $\mathrm{H}_{2} \mathrm{O}_{2}$-treated cells (see Material and Methods for details). The number of positive matches among the input sequences and the e-value from the MEME suite DREME tool are indicated above each logo. The top four logos are presented.

When we analyzed the enrichment of published Cap1-binding sites in nucleosome-free ATAC-seq peaks in $\mathrm{H}_{2} \mathrm{O}_{2}$-treated cells, we observed that ATAC-seq read signals were indeed highly increased around the Cap1 motif (Figure 4B). Importantly, a de novo motif search identified Cap1 binding sites appearing in ATAC-seq peaks that showed increased intensity after oxidative stress (Figure 4C, motif 3). Of note, some other motifs contained repetitive deoxyadenosine (poly-dA) stretches (Figure 4C, motif 1). While these might be less relevant for the recruitment of transcription factors, such signals can be explained by the preferred occurrence of poly $(\mathrm{dA}: \mathrm{dT})$ tracts in nucleosome-depleted regulatory regions, such as TATA elements upstream of the TSS, and the tendency of A-rich elements in TATA-less promoters in S. cerevisiae [86,87]. In addition, GGTTT/AAACC motifs are overrepresented in ATAC-seq data due to a binding bias of the Tn5 transposase to such regions [88]. These data suggest that a more stringent correction of the Tn5 sequence bias might be beneficial for a de novo motif discovery with higher precision. Notably, tools for advanced sequence bias correction of enzymatically prepared sequencing libraries, including DNase-seq, MNase-seq, and ATAC-seq, are available [88,89]. 
In conclusion, we here present an integrative workflow for ATAC-seq in the human fungal pathogen C. albicans. ATAC-seq offers a new robust tool to capture temporal changes in chromatin landscapes that are tightly connected to transcript abundance during fungal adaptation to stress. In addition, novel regulators that are not subject to transcriptional control at the time of sample collection might be identified based on chromatin accessibility signatures. This might aid in capturing a broader picture of highly dynamic cellular adaptations where sample drawing is limited. Finally, the combination of ATAC-seq with additional datasets has the potential to predict yet-unknown regulatory sequences and transcription factor binding sites that dictate transcriptional reprogramming and, thus, may be crucial to fungal survival upon stress encounter. Finally, the present workflow might be applicable to in vivo ATAC-seq approaches, since we were already able to obtain meaningful ATAC-seq data from as little as 50,000 C. albicans spheroplasts. The re-isolation of comparable fungal cell numbers from host model systems, such as mice, is feasible and thus, ATAC-seq might facilitate studies that elucidate fungal responses and adaptation to host defense in a tissue-specific manner. Such data will not only help to better understand fungal tissue tropism but also offer to identify new fungal genes amenable to therapeutic intervention.

Supplementary Materials: The following are available online at http://www.mdpi.com/2309-608X/6/3/182/s1, Figure S1: Number of mapped ATAC-seq reads to each C. albicans chromosome. $(\mathrm{A}, \mathrm{B})$ The aligned reads from each biological replicate of the gDNA, $\mathrm{H}_{2} \mathrm{O}_{2}$-treated $\left(\mathrm{H}_{2} \mathrm{O}_{2}\right)$ and non-treated (YPD) ATAC-seq libraries were merged and the number of mapped reads (x-axis) per chromosome (y-axis) was plotted (A). As comparison, the total size (in Mbp) of each C. albicans chromosome is presented as well (B). Figure S2: Analysis of genes with decreased chromatin accessibility in regions upstream their TSS. (A,B) Genomic regions from cluster 4 (see Figure 2C,D) were annotated to the next downstream gene and subjected to GO term enrichment analysis. Enriched biological processes are represented as dotplot (A) and cnet plot for the biological process "ribosome biogenesis" (B). The cnet plot depicts the genes associated with the presented GO term and the log2-fold change from RNA-seq data overlaid as color gradient. See figure legend from Figure 2E for detailed description. Figure S3: PCA of detected nucleosome-free ATAC-seq peaks. Called nucleosome-free ATAC-seq peaks from all samples were merged and subjected to PCA based on the presence or absence of ATAC-seq peaks in each replicate and condition (YPD and $\mathrm{H}_{2} \mathrm{O}_{2}$ ). Each dot in blue represents three biological replicates from YPD-grown cells (YPD) and each dot in red represents three biological replicates of $\mathrm{H}_{2} \mathrm{O}_{2}$-treated samples $\left(\mathrm{H}_{2} \mathrm{O}_{2}\right)$. The x-axis shows the principal component 1 (PC1) and the y-axis the principal component 2 (PC2). Table S1: Nextera primers used for ATAC-seq library preparation, Table S2: $K$-means clustering result and transcriptional regulation of associated genes, Table S3: MACS2 peak calling and edgeR analysis result, Table S4: Merged ATAC-seq and RNA-seq result, Table S5: Peaks upstream of genes with altered chromatin accessibility upon oxidative stress without changes in gene expression.

Author Contributions: S.J., M.T., and K.K. designed the experiments. S.J. and T.M. performed the experiments. M.T. and S.J. analyzed the data. M.T. established the bioinformatics workflow. S.J. and K.K. wrote the manuscript. All authors have read and agreed to the published version of the manuscript.

Funding: This work was funded by the Austrian Science Fund (FWF) project ChromFunVir (Project P32582-B) to K.K. and in part by the Austrian Science Fund (FWF) FWF-SFB-070-HIT project to K.K. M.T. was supported by an Erwin Schroedinger Return Fellowship (FWF-J3835) of the Austrian Science Fund (FWF). Open Access Funding by the Austrian Science Fund (FWF).

Acknowledgments: We are grateful to the Christoph Bock lab members Thomas Krausgruber and André Rendeiro for advice about the ATAC-seq sample preparation and bioinformatics analysis. In addition, we want to acknowledge Aaron Hernday and lab members for helpful discussions about the initial set up of ATAC-seq in C. albicans.

Conflicts of Interest: The authors declare no conflict of interest.

\section{References}

1. Rai, M.N.; Balusu, S.; Gorityala, N.; Dandu, L.; Kaur, R. Functional genomic analysis of Candida glabrata-macrophage interaction: Role of chromatin remodeling in virulence. PLoS Pathog. 2012, 8, e1002863. [CrossRef]

2. Juárez-Reyes, A.; Castaño, I. Chromatin architecture and virulence-related gene expression in eukaryotic microbial pathogens. Curr. Genet. 2019, 65, 435-443. [CrossRef] [PubMed]

3. Da Silva Dantas, A.; Lee, K.K.; Raziunaite, I.; Schaefer, K.; Wagener, J.; Yadav, B.; Gow, N.A. Cell biology of Candida albicans-host interactions. Curr. Opin. Microbiol. 2016, 34, 111-118. [CrossRef] [PubMed] 
4. López-Maury, L.; Marguerat, S.; Bähler, J. Tuning gene expression to changing environments: From rapid responses to evolutionary adaptation. Nat. Rev. Genet. 2008, 9, 583-593. [CrossRef] [PubMed]

5. Cases, I.; de Lorenzo, V.; Ouzounis, C.A. Transcription regulation and environmental adaptation in bacteria. Trends Microbiol. 2003, 11, 248-253. [CrossRef]

6. Venkatesh, S.; Workman, J.L. Histone exchange, chromatin structure and the regulation of transcription. Nat. Rev. Mol. Cell. Biol. 2015, 16, 178-189. [CrossRef]

7. Voss, T.C.; Hager, G.L. Dynamic regulation of transcriptional states by chromatin and transcription factors. Nat. Rev. Genet. 2014, 15, 69-81. [CrossRef]

8. Brown, A.J.P.; Budge, S.; Kaloriti, D.; Tillmann, A.; Jacobsen, M.D.; Yin, Z.; Ene, I.V.; Bohovych, I.; Sandai, D.; Kastora, S.; et al. Stress adaptation in a pathogenic fungus. J. Exp. Biol. 2014, 217, 144-155. [CrossRef]

9. Fisher, R.A.; Gollan, B.; Helaine, S. Persistent bacterial infections and persister cells. Nat. Rev. Microbiol. 2017, 15, 453-464. [CrossRef]

10. Wilson, D.; Thewes, S.; Zakikhany, K.; Fradin, C.; Albrecht, A.; Almeida, R.; Brunke, S.; Grosse, K.; Martin, R.; Mayer, F.; et al. Identifying infection-associated genes of Candida albicans in the postgenomic era. FEMS Yeast Res. 2009, 9, 688-700. [CrossRef]

11. Mayer, F.L.; Wilson, D.; Hube, B. Candida albicans pathogenicity mechanisms. Virulence 2013, 4, 119-128. [CrossRef] [PubMed]

12. Noble, S.M.; French, S.; Kohn, L.A.; Chen, V.; Johnson, A.D. Systematic screens of a Candida albicans homozygous deletion library decouple morphogenetic switching and pathogenicity. Nat Genet. 2010, 42, 590-598. [CrossRef] [PubMed]

13. Noble, S.M.; Gianetti, B.A.; Witchley, J.N. Candida albicans cell-type switching and functional plasticity in the mammalian host. Nat. Rev. Microbiol. 2017, 15, 96-108. [CrossRef] [PubMed]

14. Enjalbert, B.; Nantel, A.; Whiteway, M. Stress-induced gene expression in Candida albicans: Absence of a general stress response. Mol. Biol. Cell. 2002, 14, 1460-1467. [CrossRef] [PubMed]

15. Shivarathri, R.; Tscherner, M.; Zwolanek, F.; Singh, N.K.; Chauhan, N.; Kuchler, K. The fungal histone acetyl transferase Gcn 5 controls virulence of the human pathogen Candida albicans through multiple pathways. Sci. Rep. 2019, 9, 1-18. [CrossRef] [PubMed]

16. Tscherner, M.; Zwolanek, F.; Jenull, S.; Sedlazeck, F.J.; Petryshyn, A.; Frohner, I.E.; Mavrianos, J.; Chauhan, N.; von Haeseler, A.; Kuchler, K. The Candida albicans histone acetyltransferase Hat1 regulates stress resistance and virulence via distinct chromatin assembly pathways. PLoS Pathog. 2015, 11, e1005218. [CrossRef]

17. Kuchler, K.; Jenull, S.; Shivarathri, R.; Chauhan, N. Fungal KATs/KDACs: A new highway to better antifungal drugs? PLoS Pathog. 2016, 12, e1005938. [CrossRef]

18. Da Rosa, J.L.; Boyartchuk, V.L.; Zhu, L.J.; Kaufman, P.D. Histone acetyltransferase Rtt109 is required for Candida albicans pathogenesis. Proc. Natl. Acad. Sci. USA 2010, 107, 1594-1599. [CrossRef]

19. Wurtele, H.; Tsao, S.; Lépine, G.; Mullick, A.; Tremblay, J.; Drogaris, P.; Lee, E.H.; Thibault, P.; Verreault, A.; Raymond, M. Modulation of histone H3 lysine 56 acetylation as an antifungal therapeutic strategy. Nat Med. 2010, 16, 774-780. [CrossRef]

20. Hnisz, D.; Bardet, A.F.; Nobile, C.J.; Petryshyn, A.; Glaser, W.; Schöck, U.; Stark, A.; Kuchler, K. A histone deacetylase adjusts transcription kinetics at coding sequences during Candida albicans morphogenesis. PLoS Genet. 2012, 8, e1003118. [CrossRef]

21. Garnaud, C.; Champleboux, M.; Maubon, D.; Cornet, M.; Govin, J. Histone deacetylases and their inhibition in Candida species. Front. Microbiol. 2016, 7, 1-10. [CrossRef] [PubMed]

22. Robbins, N.; Wright, G.D.; Cowen, L.E. Antifungal drugs: The current armamentarium and development of new agents. In The Fungal Kingdom; ASM Press: Washington, DC, USA, 2016; pp. 903-922.

23. Lockhart, S.R. Candida auris and multidrug resistance: Defining the new normal. Fungal Genet. Biol. 2019, 131, 103243. [CrossRef] [PubMed]

24. Muñoz, J.F.; Gade, L.; Chow, N.A.; Loparev, V.N.; Juieng, P.; Berkow, E.L.; Farrer, R.A.; Litvintseva, A.P.; Cuomo, C.A. Genomic insights into multidrug-resistance, mating and virulence in Candida auris and related emerging species. Nat. Commun. 2018, 9, 5346. [CrossRef]

25. Kean, R.; Brown, J.; Gulmez, D.; Ware, A.; Ramage, G. Candida auris: A decade of understanding of an enigmatic pathogenic yeast. J. Fungi 2020, 6, 30. [CrossRef] [PubMed]

26. Lai, W.K.M.; Pugh, B.F. Understanding nucleosome dynamics and their links to gene expression and DNA replication. Nat. Rev. Mol. Cell. Biol. 2017, 18, 548-562. [CrossRef] 
27. Brogaard, K.; Xi, L.; Wang, J.-P.; Widom, J. A map of nucleosome positions in yeast at base-pair resolution. Nature 2012, 486, 496-501. [CrossRef]

28. Thurman, R.E.; Rynes, E.; Humbert, R.; Vierstra, J.; Maurano, M.T.; Haugen, E.; Sheffield, N.C.; Stergachis, A.B.; Wang, H.; Vernot, B.; et al. The accessible chromatin landscape of the human genome. Nature 2012, 489, 75-82. [CrossRef]

29. Boyle, A.P.; Davis, S.; Shulha, H.P.; Meltzer, P.; Margulies, E.H.; Weng, Z.; Furey, T.S.; Crawford, G.E. High-resolution mapping and characterization of open chromatin across the genome. Cell 2008, 132, 311-322. [CrossRef]

30. Buenrostro, J.D.; Giresi, P.G.; Zaba, L.C.; Chang, H.Y.; Greenleaf, W.J. Transposition of native chromatin for fast and sensitive epigenomic profiling of open chromatin, DNA-binding proteins and nucleosome position. Nat. Methods 2013, 10, 1213-1218. [CrossRef]

31. Schep, A.N.; Buenrostro, J.D.; Denny, S.K.; Schwartz, K.; Sherlock, G.; Greenleaf, W.J. Structured nucleosome fingerprints enable high-resolution mapping of chromatin architecture within regulatory regions. Genome Res. 2015, 25, 1757-1770. [CrossRef]

32. Lu, Z.; Hofmeister, B.T.; Vollmers, C.; DuBois, R.M.; Schmitz, R.J. Combining ATAC-seq with nuclei sorting for discovery of cis-regulatory regions in plant genomes. Nucleic Acids Res. 2017, 45, 1-13. [CrossRef] [PubMed]

33. Wu, J.; Huang, B.; Chen, H.; Yin, Q.; Liu, Y.; Xiang, Y.; Zhang, B.; Liu, B.; Wang, Q.; Xia, W.; et al. The landscape of accessible chromatin in mammalian preimplantation embryos. Nature 2016, 534, 652-657. [CrossRef]

34. Maezawa, S.; Yukawa, M.; Alavattam, K.G.; Barski, A.; Namekawa, S.H. Dynamic reorganization of open chromatin underlies diverse transcriptomes during spermatogenesis. Nucleic Acids Res. 2017, 46, 593-608. [CrossRef]

35. Liu, C.; Wang, M.; Wei, X.; Wu, L.; Xu, J.; Dai, X.; Xia, J.; Cheng, M.; Yuan, Y.; Zhang, P.; et al. An ATAC-seq atlas of chromatin accessibility in mouse tissues. Sci. Data 2019, 6, 65. [CrossRef] [PubMed]

36. Corces, M.R.; Trevino, A.E.; Hamilton, E.G.; Greenside, P.G.; Sinnott-Armstrong, N.A.; Vesuna, S.; Satpathy, A.T.; Rubin, A.J.; Montine, K.S.; Wu, B.; et al. An improved ATAC-seq protocol reduces background and enables interrogation of frozen tissues. Nat. Methods 2017, 14, 959-962. [CrossRef] [PubMed]

37. Buenrostro, J.D.; Wu, B.; Litzenburger, U.M.; Ruff, D.; Gonzales, M.L.; Snyder, M.P.; Chang, H.Y.; Greenleaf, W.J. Single-cell chromatin accessibility reveals principles of regulatory variation. Nature 2015, 523, 486-490. [CrossRef] [PubMed]

38. Lareau, C.A.; Duarte, F.M.; Chew, J.G.; Kartha, V.K.; Burkett, Z.D.; Kohlway, A.S.; Pokholok, D.; Aryee, M.J.; Steemers, F.J.; Lebofsky, R.; et al. Droplet-based combinatorial indexing for massive-scale single-cell chromatin accessibility. Nat. Biotechnol. 2019, 37, 916-924. [CrossRef] [PubMed]

39. Toenhake, C.G.; Fraschka, S.A.K.; Vijayabaskar, M.S.; Westhead, D.R.; van Heeringen, S.J.; Bártfai, R. Chromatin accessibility-based characterization of the gene regulatory network underlying plasmodium falciparum blood-stage development. Cell Host Microbe 2018, 23, 557-569. [CrossRef]

40. Rogerson, C.; Britton, E.; Withey, S.; Hanley, N.; Ang, Y.S.; Sharrocks, A.D. Identification of a primitive intestinal transcription factor network shared between esophageal adenocarcinoma and its precancerous precursor state. Genome Res. 2019, 29, 723-736. [CrossRef]

41. Li, Z.; Schulz, M.H.; Look, T.; Begemann, M.; Zenke, M.; Costa, I.G. Identification of transcription factor binding sites using ATAC-seq. Genome Biol. 2019, 20, 45. [CrossRef]

42. Liu, L.; Leng, L.; Liu, C.; Lu, C.; Yuan, Y.; Wu, L.; Gong, F.; Zhang, S.; Wei, X.; Wang, M.; et al. An integrated chromatin accessibility and transcriptome landscape of human pre-implantation embryos. Nat. Commun. 2019, 10, 364. [CrossRef] [PubMed]

43. Enjalbert, B.; MacCallum, D.M.; Odds, F.C.; Brown, A.J.P. Niche-specific activation of the oxidative stress response by the pathogenic fungus Candida albicans. Infect. Immun. 2007, 75, 2143-2151. [CrossRef] [PubMed]

44. Kaloriti, D.; Jacobsen, M.; Yin, Z.; Patterson, M.; Tillmann, A.; Smith, D.A.; Cook, E.; You, T.; Grimm, M.J.; Bohovych, I.; et al. Mechanisms underlying the exquisite sensitivity of Candida albicans to combinatorial cationic and oxidative stress that enhances the potent fungicidal activity of phagocytes. MBio 2014, 5, e01334-14. [CrossRef] [PubMed]

45. Znaidi, S.; Barker, K.S.; Weber, S.; Alarco, A.-M.; Liu, T.T.; Boucher, G.; Rogers, P.D.; Raymond, M. Identification of the Candida albicans Cap1p regulon. Eukaryot. Cell 2009, 8, 806-820. [CrossRef] [PubMed] 
46. Gillum, A.M.; Tsay, E.Y.H.; Kirsch, D.R. Isolation of the Candida albicans gene for orotidine-5'-phosphate decarboxylase by complementation of S. cerevisiae ura3 and E. coli pyrF mutations. MGG Mol. Gen. Genet. 1984, 198, 179-182. [CrossRef]

47. Goryshin, I.Y.; Miller, J.A.; Kil, Y.V.; Lanzov, V.A.; Reznikoff, W.S. Tn5/IS50 target recognition. Proc. Natl. Acad. Sci. USA 1998, 95, 10716-10721. [CrossRef]

48. Hoffman, C.S.; Winston, F. A ten-minute DNA preparation from yeast efficiently releases autonomous plasmids for transformaion of Escherichia coli. Gene 1987, 57, 267-272. [CrossRef]

49. Buenrostro, J.D.; Wu, B.; Chang, H.Y.; Greenleaf, W.J. ATAC-seq: A method for assaying chromatin accessibility genome-wide. Curr. Protoc. Mol. Biol. 2015, 109, 21-29. [CrossRef]

50. Andrews, S. FastQC: A Quality Control Tool for High Throughput Sequence Data; Babraham Institute: Cambridge, UK, 2010.

51. Sedlazeck, F.J.; Rescheneder, P.; von Haeseler, A. NextGenMap: Fast and accurate read mapping in highly polymorphic genomes. Bioinformatics 2013, 29, 2790-2791. [CrossRef]

52. Ramírez, F.; Ryan, D.P.; Grüning, B.; Bhardwaj, V.; Kilpert, F.; Richter, A.S.; Heyne, S.; Dündar, F.; Manke, T. deepTools2: A next generation web server for deep-sequencing data analysis. Nucleic Acids Res. 2016, 44, W160-W165.

53. Robinson, J.T.; Thorvaldsdóttir, H.; Winckler, W.; Guttman, M.; Lander, E.S.; Getz, G.; Mesirov, J.P. Integrative genomics viewer. Nat. Biotechnol. 2011, 29, 24-26. [CrossRef]

54. Lawrence, M.; Huber, W.; Pagès, H.; Aboyoun, P.; Carlson, M.; Gentleman, R.; Morgan, M.T.; Carey, V.J. Software for computing and annotating genomic ranges. PLoS Comput. Biol. 2013, 9, e1003118. [CrossRef] [PubMed]

55. Tsankov, A.M.; Thompson, D.A.; Socha, A.; Regev, A.; Rando, O.J. The role of nucleosome positioning in the evolution of gene regulation. PLoS Biol. 2010, 8, e1000414. [CrossRef] [PubMed]

56. Yu, G.; Wang, L.-G.; Han, Y.; He, Q.-Y. ClusterProfiler: An R package for comparing biological themes among gene clusters. OMISC: J. Integr. Biol. 2012, 16, 284-287. [CrossRef] [PubMed]

57. Zhang, Y.; Liu, T.; Meyer, C.A.; Eeckhoute, J.; Johnson, D.S.; Bernstein, B.E.; Nusbaum, C.; Myers, R.M.; Brown, M.; Li, W.; et al. Model-based analysis of ChIP-Seq (MACS). Genome Biol. 2008, 9, R137. [CrossRef]

58. Li, H.; Handsaker, B.; Wysoker, A.; Fennell, T.; Ruan, J.; Homer, N.; Marth, G.; Abecasis, G.; Durbin, R.; 1000 Genome Project Data Processing Subgroup. The sequence alignment/map format and SAMtools. Bioinformatics 2009, 25, 2078-2079. [CrossRef]

59. Anders, S.; Pyl, P.T.; Huber, W. HTSeq-A Python framework to work with high-throughput sequencing data. Bioinformatics 2014, 31, 166-169. [CrossRef]

60. Yu, G.; Wang, L.-G.; He, Q.-Y. ChIPseeker: An R/Bioconductor package for ChIP peak annotation, comparison and visualization. Bioinformatics 2015, 31, 2382-2383. [CrossRef]

61. Gel, B.; Serra, E. karyoploteR: An R/Bioconductor package to plot customizable genomes displaying arbitrary data. Bioinformatics 2017, 33, 3088-3090. [CrossRef]

62. Robinson, M.D.; McCarthy, D.J.; Smyth, G.K. edgeR: A Bioconductor package for differential expression analysis of digital gene expression data. Bioinformatics 2009, 26, 139-140. [CrossRef]

63. Bailey, T.L.; Boden, M.; Buske, F.A.; Frith, M.; Grant, C.E.; Clementi, L.; Ren, J.; Li, W.W.; Noble, W.S. MEME Suite: Tools for motif discovery and searching. Nucleic Acids Res. 2009, 37 (Suppl. S2), W202-W208. [CrossRef] [PubMed]

64. Grant, C.E.; Bailey, T.L.; Noble, W.S. FIMO: Scanning for occurrences of a given motif. Bioinformatics 2011, 27, 1017-1018. [CrossRef] [PubMed]

65. Bailey, T.L. DREME: Motif discovery in transcription factor ChIP-seq data. Bioinformatics 2011, 27, $1653-1659$. [CrossRef] [PubMed]

66. Wickham, H. ggplot2: Elegant Graphics for Data Analysis; Springer: New York, NY, USA, 2016.

67. Adey, A.; Morrison, H.G.; Asan Xun, X.; Kitzman, J.O.; Turner, E.H.; Stackhouse, B.; MacKenzie, A.P.; Caruccio, N.C.; Zhang, X.; Shendure, J. Rapid, low-input, low-bias construction of shotgun fragment libraries by high-density in vitro transposition. Genome Biol. 2010, 11, R119. [CrossRef]

68. Bai, L.; Morozov, A.V. Gene regulation by nucleosome positioning. Trends Genet. 2010, 26, 476-483. [CrossRef]

69. Cottier, F.; Tan, A.S.M.; Chen, J.; Lum, J.; Zolezzi, F.; Poidinger, M.; Pavelka, N. The transcriptional stress response of Candida albicans to weak organic acids. G3 Genes Genomes Genet. 2015, 5, 497-505. 
70. Lorenz, M.C.; Bender, J.A.; Fink, G.R. Transcriptional response of Candida albicans upon internalization by macrophages. Eukaryot. Cell. 2004, 3, 1076-1087. [CrossRef]

71. Enjalbert, B.; Smith, D.A.; Cornell, M.J.; Alam, I.; Nicholls, S.; Brown, A.J.P.; Quinn, J. Role of the Hog1 stress-activated protein kinase in the global transcriptional response to stress in the fungal pathogen Candida albicans. Mol. Biol. Cell. 2006, 17, 1018-1032. [CrossRef]

72. Gasch, A.P.; Spellman, P.T.; Kao, C.M.; Carmel-Harel, O.; Eisen, M.B.; Storz, G.; Botstein, D.; Brown, P.O. Genomic expression programs in the response of yeast cells to environmental changes. Mol. Biol. Cell. 2000, 11, 4241-4257. [CrossRef]

73. Albert, B.; Kos-Braun, I.C.; Henras, A.K.; Dez, C.; Rueda, M.P.; Zhang, X.; Gadal, O.; Kos, M.; Shore, D. A ribosome assembly stress response regulates transcription to maintain proteome homeostasis. Elife 2019, 8, e45002. [CrossRef]

74. Klemm, S.L.; Shipony, Z.; Greenleaf, W.J. Chromatin accessibility and the regulatory epigenome. Nat. Rev. Genet. 2019, 20, 207-220. [CrossRef] [PubMed]

75. Hoffman, M.M.; Ernst, J.; Wilder, S.P.; Kundaje, A.; Harris, R.S.; Libbrecht, M.; Giardine, B.; Ellenbogen, P.M.; Bilmes, J.A.; Birney, E.; et al. Integrative annotation of chromatin elements from ENCODE data. Nucleic Acids Res. 2013, 41, 827-841. [CrossRef] [PubMed]

76. Deaton, A.M.; Gómez-Rodríguez, M.; Mieczkowski, J.; Tolstorukov, M.Y.; Kundu, S.; Sadreyev, R.I.; Jansen, L.E.; Kingston, R.E. Enhancer regions show high histone H3.3 turnover that changes during differentiation. Elife 2016, 5, 1-24. [CrossRef] [PubMed]

77. Hendrickson, D.G.; Soifer, I.; Wranik, B.J.; Botstein, D.; Scott McIsaac, R. Simultaneous profiling of DNA accessibility and gene expression dynamics with ATAC-seq and RNA-seq. Methods Mol. Biol. 2018, 1819, 317-333.

78. Wysong, D.R.; Christin, L.; Sugar, A.M.; Robbins, P.W.; Diamond, R.D. Cloning and sequencing of a Candida albicans catalase gene and effects of disruption of this gene. Infect. Immun. 1998, 66, 1953-1961. [CrossRef]

79. Wang, Y.; Cao, Y.Y.; Jia, X.M.; Cao, Y.B.; Gao, P.H.; Fu, X.P.; Ying, K.; Chen, W.S.; Jiang, Y.Y. Cap1p is involved in multiple pathways of oxidative stress response in Candida albicans. Free Radic. Biol. Med. 2006, 40, 1201-1209. [CrossRef]

80. Piekarska, K.; Hardy, G.; Mol, E.; van den Burg, J.; Strijbis, K.; van Roermund, C.; van den Berg, M.; Distel, B. The activity of the glyoxylate cycle in peroxisomes of Candida albicans depends on a functional beta-oxidation pathway: Evidence for reduced metabolite transport across the peroxisomal membrane. Microbiology 2008, 154, 3061-3072. [CrossRef]

81. Yan, F.; Powell, D.R.; Curtis, D.J.; Wong, N.C. From reads to insight: A hitchhiker's guide to ATAC-seq data analysis. Genome Biol. 2020, 21, 22. [CrossRef]

82. Urban, C.; Xiong, X.; Sohn, K.; Schröppel, K.; Brunner, H.; Rupp, S. The moonlighting protein Tsa1p is implicated in oxidative stress response and in cell wall biogenesis in Candida albicans. Mol. Microbiol. 2005, 57, 1318-1341. [CrossRef]

83. Lee, K.; Kim, S.C.; Jung, I.; Kim, K.; Seo, J.; Lee, H.-S.; Bogu, G.K.; Kim, D.; Lee, S.; Lee, B.; et al. Genetic landscape of open chromatin in yeast. PLoS Genet. 2013, 9, e1003229. [CrossRef]

84. Hernday, A.D.; Lohse, M.B.; Fordyce, P.M.; Nobile, C.J.; Derisi, J.L.; Johnson, A.D. Structure of the transcriptional network controlling white-opaque switching in Candida albicans. Mol. Microbiol. 2013, 90, 22-35.

85. Nobile, C.J.; Fox, E.P.; Nett, J.E.; Sorrells, T.R.; Mitrovich, Q.M.; Hernday, A.D.; Tuch, B.B.; Andes, D.R.; Johnson, A.D. A recently evolved transcriptional network controls biofilm development in Candida albicans. Cell 2012, 148, 126-138. [CrossRef]

86. Segal, E.; Widom, J. Poly(dA:dT) tracts: Major determinants of nucleosome organization. Curr. Opin. Struct. Biol. 2009, 19, 65-71. [CrossRef]

87. Rhee, H.S.; Pugh, B.F. Genome-wide structure and organization of eukaryotic pre-initiation complexes. Nature 2012, 483, 295-301. [CrossRef] 
88. Wang, J.R.; Quach, B.; Furey, T.S. Correcting nucleotide-specific biases in high-throughput sequencing data. BMC Bioinformatics 2017, 18, 357. [CrossRef]

89. Martins, A.L.; Walavalkar, N.M.; Anderson, W.D.; Zang, C.; Guertin, M.J. Universal correction of enzymatic sequence bias reveals molecular signatures of protein/DNA interactions. Nucleic Acids Res. 2017, 46, e9. [CrossRef] 